\title{
Partial Correctness of GCD Algorithm
}

\author{
Ievgen Ivanov \\ Taras Shevchenko National University \\ Kyiv, Ukraine
}

\author{
Artur Korniłowicz (iD \\ Institute of Informatics \\ University of Białystok \\ Poland
}

\author{
Mykola Nikitchenkc \\ Taras Shevchenko National University \\ Kyiv, Ukraine
}

\begin{abstract}
Summary. In this paper we present a formalization in the Mizar system 2. 1. of the correctness of the subtraction-based version of Euclid's algorithm computing the greatest common divisor of natural numbers. The algorithm is written in terms of simple-named complex-valued nominative data [11, 4].

The validity of the algorithm is presented in terms of semantic Floyd-Hoare triples over such data [7. Proofs of the correctness are based on an inference system for an extended Floyd-Hoare logic with partial pre- and post-conditions [8, 10, 5, 3.
\end{abstract}

$\begin{array}{lllll}\text { MSC: 68Q60 68T37 03B70 03B35 } & \text { 63 }\end{array}$

Keywords: greatest common divisor; nominative data; program verification

MML identifier: NOMIN_4, version: 8.1.08 5.53.1335

From now on $v$ denotes an object, $V, A$ denote sets, and $f$ denotes a binominative function over simple-named complex-valued nominative data of $V$ and $A$.

Let us consider $A$. We say that $A$ is complex containing if and only if (Def. 1) $\mathbb{C} \subseteq A$.

One can verify that there exists a set which is complex containing and every set which is complex containing is also non empty.

The scheme BinPredToFunEx deals with sets $\mathcal{X}, \mathcal{Y}$ and a binary predicate $\mathcal{P}$ and states that 
(Sch. 1) There exists a function $f$ from $\mathcal{X} \times \mathcal{Y}$ into Boolean such that for every objects $x, y$ such that $x, y \in \mathcal{Y}$ holds if $\mathcal{P}[x, y]$, then $f(x, y)=$ true and if not $\mathcal{P}[x, y]$, then $f(x, y)=$ false.

The scheme BinPredToFunUnique deals with sets $\mathcal{X}, \mathcal{Y}$ and a binary predicate $\mathcal{P}$ and states that

(Sch. 2) For every functions $f, g$ from $\mathcal{X} \times \mathcal{Y}$ into Boolean such that for every objects $x, y$ such that $x, y \in \mathcal{Y}$ holds if $\mathcal{P}[x, y]$, then $f(x, y)=$ true and if not $\mathcal{P}[x, y]$, then $f(x, y)=$ false and for every objects $x, y$ such that $x, y \in \mathcal{Y}$ holds if $\mathcal{P}[x, y]$, then $g(x, y)=$ true and if not $\mathcal{P}[x, y]$, then $g(x, y)=$ false holds $f=g$.

The scheme Lambda2Unique deals with sets $\mathcal{X}, \mathcal{Y}, \mathcal{Z}$ and a binary functor $\mathcal{F}$ yielding an object and states that

(Sch. 3) For every functions $f, g$ from $\mathcal{X} \times \mathcal{Y}$ into $\mathcal{Z}$ such that for every objects $x, y$ such that $x, y \in \mathcal{Y}$ holds $f(x, y)=\mathcal{F}(x, y)$ and for every objects $x, y$ such that $x, y \in \mathcal{Y}$ holds $g(x, y)=\mathcal{F}(x, y)$ holds $f=g$.

Let us consider $V$ and $A$. The functor nonatomicsND $(V, A)$ yielding a set is defined by the term

(Def. 2) the set of all $d$ where $d$ is a non-atomic nominative data of $V$ and $A$.

Now we state the propositions:

(1) Let us consider an object $d$. Suppose $d \in$ nonatomicsND $(V, A)$. Then $d$ is a non-atomic nominative data of $V$ and $A$.

(2) $\emptyset \in$ nonatomicsND $(V, A)$.

Let us consider $V$ and $A$. One can verify that nonatomicsND $(V, A)$ is non empty and functional.

We say that $V$ is without nonatomic nominative data w.r.t. $A$ if and only if

(Def. 3) $A$ misses nonatomicsND $(V, A)$.

Now we state the propositions:

(3) If $V$ is without nonatomic nominative data w.r.t. $A$, then for every nonatomic nominative data $d$ of $V$ and $A, d \notin A$.

(4) Suppose $V$ is without nonatomic nominative data w.r.t. $A$ and $v \in V$. Let us consider a non-atomic nominative data $d_{1}$ of $V$ and $A$, and a nominative data $d_{2}$ with simple names from $V$ and complex values from $A$. Then $\operatorname{dom}\left(d_{1} \nabla_{a}^{v} d_{2}\right)=\{v\} \cup \operatorname{dom} d_{1}$. The theorem is a consequence of (3).

(5) Suppose $V$ is without nonatomic nominative data w.r.t. $A$. Let us consider a non-atomic nominative data $d$ of $V$ and $A$. Suppose $v \in V$ and $d \in \operatorname{dom} f$. Then $\operatorname{dom}\left(\left(\operatorname{Asg}^{v}(f)\right)(d)\right)=\operatorname{dom} d \cup\{v\}$. The theorem is a consequence of (3). 
In the sequel $d$ denotes a nominative data with simple names from $V$ and complex values from $A$.

(6) Let us consider a non-atomic nominative data $d_{1}$ of $V$ and $A$. Suppose $v \in V$ and $V$ is without nonatomic nominative data w.r.t. $A$. Then $d_{1} \nabla_{a}^{v} d \in \operatorname{dom}\left(v \Rightarrow_{a}\right)$. The theorem is a consequence of (4).

From now on $a, b, c, x, y, z$ denote elements of $V$ and $p, q, r, s$ denote partial predicates over simple-named complex-valued nominative date of $V$ and $A$.

Let us consider $V, A, d$, and $a$. We say that $d$ is an extended real on $a$ if and only if

(Def. 4) $\left(a \Rightarrow_{a}\right)(d)$ is extended real.

We say that $d$ is a complex on $a$ if and only if

(Def. 5) $\left(a \Rightarrow_{a}\right)(d)$ is complex.

We say that $d$ is a value on $a$ if and only if

(Def. 6) $\left(a \Rightarrow_{a}\right)(d) \in A$.

Now we state the propositions:

(7) If $A$ is complex containing and for every $d, d$ is a complex on $a$, then for every $d, d$ is a value on $a$.

(8) If for every $d, d$ is a value on $a$, then $\operatorname{rng} a \Rightarrow_{a} \subseteq A$.

(9) If for every $d, d$ is a value on $a$ and for every $d, d$ is a value on $b$, then $\operatorname{rng}\left\langle a \Rightarrow_{a}, b \Rightarrow_{a}\right\rangle \subseteq A \times A$. The theorem is a consequence of (8).

Let us consider $V$ and $A$. Let $a, b$ be elements of $V$ and $p$ be a function from $A \times A$ into Boolean. The functor lift-binary-pred $(p, a, b)$ yielding a partial predicate over simple-named complex-valued nominative data of $V$ and $A$ is defined by the term

(Def. 7) $p \cdot\left\langle a \Rightarrow_{a}, b \Rightarrow_{a}\right\rangle$.

Let $o_{1}$ be a function from $A \times A$ into $A$. The functor lift-binary-op $\left(o_{1}, a, b\right)$ yielding a binominative function over simple-named complex-valued nominative data of $V$ and $A$ is defined by the term

(Def. 8) $o_{1} \cdot\left\langle a \Rightarrow_{a}, b \Rightarrow_{a}\right\rangle$.

The functor Equality $(A)$ yielding a function from $A \times A$ into Boolean is defined by

(Def. 9) for every objects $a, b$ such that $a, b \in A$ holds if $a=b$, then $i t(a, b)=$ true and if $a \neq b$, then $i t(a, b)=$ false.

Let us consider $V$. Let $x, y$ be elements of $V$. The functor $\operatorname{Equality}(A, x, y)$ yielding a partial predicate over simple-named complex-valued nominative data of $V$ and $A$ is defined by the term

(Def. 10) lift-binary-pred(Equality $(A), x, y)$. 
Let $x, y$ be objects. We say that $x$ is less than $y$ if and only if

(Def. 11) there exist extended reals $x_{1}, y_{1}$ such that $x_{1}=x$ and $y_{1}=y$ and $x_{1}<y_{1}$.

Observe that the predicate is irreflexive and asymmetric.

Now we state the proposition:

(10) Let us consider extended reals $x, y$. If $x$ is not less than $y$, then $y$ is less than $x$ or $x=y$.

Let us consider $A$. The functor $\operatorname{less}(A)$ yielding a function from $A \times A$ into Boolean is defined by

(Def. 12) for every objects $x, y$ such that $x, y \in A$ holds if $x$ is less than $y$, then it $(x, y)=$ true and if $x$ is not less than $y$, then $i t(x, y)=$ false.

Let us consider $V$. Let $x, y$ be elements of $V$. The functor $\operatorname{less}(A, x, y)$ yielding a partial predicate over simple-named complex-valued nominative data of $V$ and $A$ is defined by the term

(Def. 13) lift-binary-pred $(\operatorname{less}(A), x, y)$.

Now we state the propositions:

(11) Suppose for every $d, d$ is a value on $a$ and for every $d, d$ is a value on $b$. Then $\operatorname{dom}(\operatorname{Equality}(A, a, b))=\operatorname{dom}\left(a \Rightarrow_{a}\right) \cap \operatorname{dom}\left(b \Rightarrow_{a}\right)$. The theorem is a consequence of (9).

(12) Suppose for every $d, d$ is a value on $a$ and for every $d, d$ is a value on $b$. Then $\operatorname{dom}(\operatorname{less}(A, a, b))=\operatorname{dom}\left(a \Rightarrow_{a}\right) \cap \operatorname{dom}\left(b \Rightarrow_{a}\right)$. The theorem is a consequence of $(9)$.

(13) Suppose for every $d, d$ is a value on $a$ and for every $d, d$ is a value on $b$ and for every $d, d$ is an extended real on $a$ and for every $d, d$ is an extended real on $b$. Then $\neg \operatorname{Equality}(A, a, b)=\operatorname{less}(A, a, b) \vee \operatorname{less}(A, b, a)$.

(14) Suppose for every $d, d$ is a value on $a$ and for every $d, d$ is a value on $b$ and $d$ is an extended real on $a$ and $d$ is an extended real on $b$ and $d \in \operatorname{dom}(\neg \operatorname{Equality}(A, a, b))$ and $(\neg \operatorname{Equality}(A, a, b))(d)=$ true. Then

(i) $d \in \operatorname{dom}(\operatorname{less}(A, a, b))$ and $(\operatorname{less}(A, a, b))(d)=$ true, or

(ii) $d \in \operatorname{dom}(\operatorname{less}(A, b, a))$ and $(\operatorname{less}(A, b, a))(d)=$ true.

The theorem is a consequence of (10) and (12).

Let $x, y$ be objects. Assume $x$ is a complex number and $y$ is a complex number. The functor $x-y$ yielding a complex number is defined by

(Def. 14) there exist complex numbers $x_{1}, y_{1}$ such that $x_{1}=x$ and $y_{1}=y$ and it $=x_{1}-y_{1}$.

Let us consider $A$. Assume $A$ is complex containing. The functor subtraction $A$ yielding a function from $A \times A$ into $A$ is defined by 
(Def. 15) for every objects $x, y$ such that $x, y \in A$ holds $i t(x, y)=x-y$.

Let us consider $V$. Let $x, y$ be elements of $V$. The functor $\operatorname{subtraction}(A, x, y)$ yielding a binominative function over simple-named complex-valued nominative data of $V$ and $A$ is defined by the term

(Def. 16) lift-binary-op(subtraction $A, x, y)$.

Let us consider $a$ and $b$. The functor gcd-conditional-step $(V, A, a, b)$ yielding a binominative function over simple-named complex-valued nominative data of $V$ and $A$ is defined by the term

(Def. 17) $\operatorname{IF}\left(\operatorname{less}(A, b, a), \operatorname{Asg}^{a}(\operatorname{subtraction}(A, a, b)), \operatorname{id}_{\mathrm{PP}}\left(\mathrm{ND}_{\mathrm{SC}}(V, A)\right)\right)$.

The functor gcd-loop-body $(V, A, a, b)$ yielding a binominative function over simple-named complex-valued nominative data of $V$ and $A$ is defined by the term

(Def. 18) gcd-conditional-step $(V, A, a, b) \bullet \operatorname{gcd}$-conditional-step $(V, A, b, a)$.

The functor gcd-main-loop $(V, A, a, b)$ yielding a binominative function over simple-named complex-valued nominative data of $V$ and $A$ is defined by the term

(Def. 19) $\mathrm{WH}(\neg \operatorname{Equality}(A, a, b), \operatorname{gcd}-\operatorname{loop}-\operatorname{body}(V, A, a, b))$.

Let us consider $x$ and $y$. The functor gcd-var-init $(V, A, a, b, x, y)$ yielding a binominative function over simple-named complex-valued nominative data of $V$ and $A$ is defined by the term

(Def. 20) $\operatorname{Asg}^{a}\left(x \Rightarrow_{a}\right) \bullet \operatorname{Asg}^{b}\left(y \Rightarrow_{a}\right)$.

The functor gcd-main-part $(V, A, a, b, x, y)$ yielding a binominative function over simple-named complex-valued nominative data of $V$ and $A$ is defined by the term

(Def. 21) gcd-var-init $(V, A, a, b, x, y) \bullet \operatorname{gcd}-m a i n-\operatorname{loop}(V, A, a, b)$.

Let us consider $z$. The functor $\operatorname{gcd}-\operatorname{program}(V, A, a, b, x, y, z)$ yielding a binominative function over simple-named complex-valued nominative data of $V$ and $A$ is defined by the term

(Def. 22) gcd-main-part $(V, A, a, b, x, y) \bullet \operatorname{Asg}^{z}\left(a \Rightarrow_{a}\right)$.

From now on $x_{0}, y_{0}$ denote natural numbers.

Let us consider $V, A, x, y, x_{0}$, and $y_{0}$. Let $d$ be an object. We say that $x_{0}$, $y_{0}$ and $d$ constitute a valid input for the gcd w.r.t. $V, A, x$ and $y$ if and only if

(Def. 23) there exists a non-atomic nominative data $d_{1}$ of $V$ and $A$ such that $d=d_{1}$ and $x, y \in \operatorname{dom} d_{1}$ and $d_{1}(x)=x_{0}$ and $d_{1}(y)=y_{0}$.

The functor valid-gcd-input $\left(V, A, x, y, x_{0}, y_{0}\right)$ yielding a partial predicate over simple-named complex-valued nominative data of $V$ and $A$ is defined by 
(Def. 24) $\operatorname{dom} i t=\mathrm{ND}_{\mathrm{SC}}(V, A)$ and for every object $d$ such that $d \in \operatorname{dom} i t$ holds if $x_{0}, y_{0}$ and $d$ constitute a valid input for the gcd w.r.t. $V, A, x$ and $y$, then $i t(d)=$ true and if $x_{0}, y_{0}$ and $d$ do not constitute a valid input for the ged w.r.t. $V, A, x$ and $y$, then $i t(d)=$ false.

One can check that valid-gcd-input $\left(V, A, x, y, x_{0}, y_{0}\right)$ is total.

Let us consider $z$. Let $d$ be an object. We say that $x_{0}, y_{0}$ and $d$ constitute a valid output for the ged w.r.t. $V, A$ and $z$ if and only if

(Def. 25) there exists a non-atomic nominative data $d_{1}$ of $V$ and $A$ such that $d=d_{1}$ and $z \in \operatorname{dom} d_{1}$ and $d_{1}(z)=\operatorname{gcd}\left(x_{0}, y_{0}\right)$.

The functor valid-gcd-output $\left(V, A, z, x_{0}, y_{0}\right)$ yielding a partial predicate over simple-named complex-valued nominative data of $V$ and $A$ is defined by

(Def. 26) $\operatorname{dom} i t=\{d$, where $d$ is a nominative data with simple names from $V$ and complex values from $\left.A: d \in \operatorname{dom}\left(z \Rightarrow_{a}\right)\right\}$ and for every object $d$ such that $d \in \operatorname{dom} i t$ holds if $x_{0}, y_{0}$ and $d$ constitute a valid output for the gcd w.r.t. $V, A$ and $z$, then $i t(d)=$ true and if $x_{0}, y_{0}$ and $d$ do not constitute a valid output for the gcd w.r.t. $V, A$ and $z$, then $i t(d)=$ false.

Let us consider $a$ and $b$. Let $d$ be an object. We say that $x_{0}, y_{0}$ and $d$ constitute a valid invariant for the ged w.r.t. $V, A, a$ and $b$ if and only if

(Def. 27) there exists a non-atomic nominative data $d_{1}$ of $V$ and $A$ such that $d=d_{1}$ and $a, b \in \operatorname{dom} d_{1}$ and there exist natural numbers $x, y$ such that $x=d_{1}(a)$ and $y=d_{1}(b)$ and $\operatorname{gcd}(x, y)=\operatorname{gcd}\left(x_{0}, y_{0}\right)$.

The functor gcd-inv $\left(V, A, a, b, x_{0}, y_{0}\right)$ yielding a partial predicate over simplenamed complex-valued nominative data of $V$ and $A$ is defined by

(Def. 28) $\operatorname{dom} i t=\mathrm{ND}_{\mathrm{SC}}(V, A)$ and for every object $d$ such that $d \in \operatorname{dom} i t$ holds if $x_{0}, y_{0}$ and $d$ constitute a valid invariant for the gcd w.r.t. $V, A, a$ and $b$, then $i t(d)=$ true and if $x_{0}, y_{0}$ and $d$ do not constitute a valid invariant for the gcd w.r.t. $V, A, a$ and $b$, then $i t(d)=$ false.

Observe that $\operatorname{gcd}-\operatorname{inv}\left(V, A, a, b, x_{0}, y_{0}\right)$ is total.

Now we state the propositions:

$\left\langle\sim \mathrm{SP}_{\mathrm{P}}\left(p, x \Rightarrow_{a}, a\right), \operatorname{Asg}^{a}\left(x \Rightarrow_{a}\right), p\right\rangle$ is an $\operatorname{SFHT} \operatorname{ND}_{\mathrm{SC}}(V, A)$.

(16) Suppose $V$ is not empty and $V$ is without nonatomic nominative data w.r.t. $A$ and $a \neq b$ and $a \neq y$.

Then 〈valid-gcd-input $\left(V, A, x, y, x_{0}, y_{0}\right)$, gcd-var-init $(V, A, a, b, x, y)$, gcd-inv $\left.\left(V, A, a, b, x_{0}, y_{0}\right)\right\rangle$ is an $\operatorname{SFHT} \mathrm{ND}_{\mathrm{SC}}(V, A)$.

Proof: Set $D_{3}=x \Rightarrow_{a}$. Set $D_{4}=y \Rightarrow_{a}$. Set $p=\operatorname{gcd}-i n v\left(V, A, a, b, x_{0}, y_{0}\right)$. Set $Q=\mathrm{S}_{\mathrm{P}}\left(p, D_{4}, b\right)$. Set $P=\mathrm{S}_{\mathrm{P}}\left(Q, D_{3}, a\right)$. Set $G=\operatorname{Asg}^{b}\left(D_{4}\right)$. Set $I=$ valid-gcd-input $\left(V, A, x, y, x_{0}, y_{0}\right) .\langle\sim Q, G, p\rangle$ is an $\operatorname{SFHT}_{\text {of }} \operatorname{ND}_{\mathrm{SC}}(V, A)$. $I \models P$. 
(17) Suppose $V$ is not empty and $V$ is without nonatomic nominative data w.r.t. $A$ and $a \neq b$ and $A$ is complex containing and for every $d, d$ is a complex on $a$ and for every $d, d$ is a complex on $b$.

Then $\left\langle\operatorname{less}(A, b, a) \wedge \operatorname{gcd}-\operatorname{inv}\left(V, A, a, b, x_{0}, y_{0}\right), \operatorname{Asg}^{a}(\operatorname{subtraction}(A, a, b))\right.$, $\left.\operatorname{gcd}-\operatorname{inv}\left(V, A, a, b, x_{0}, y_{0}\right)\right\rangle$ is an $\operatorname{SFHT}_{\text {of }} \mathrm{ND}_{\mathrm{SC}}(V, A)$.

Proof: Set $i=\operatorname{gcd}-\operatorname{inv}\left(V, A, a, b, x_{0}, y_{0}\right)$. Set $l=\operatorname{less}(A, b, a)$. Set $D=$ $\operatorname{subtraction}(A, a, b)$. Set $f=\operatorname{Asg}^{a}(D)$. Set $p=l \wedge i$. For every $d$ such that $d \in \operatorname{dom} p$ and $p(d)=$ true and $d \in \operatorname{dom} f$ and $f(d) \in \operatorname{dom} i$ holds $i(f(d))=$ true.

(18) Suppose $V$ is not empty and $V$ is without nonatomic nominative data w.r.t. $A$ and $a \neq b$ and $A$ is complex containing and for every $d, d$ is a complex on $a$ and for every $d, d$ is a complex on $b$.

Then $\left\langle\operatorname{less}(A, a, b) \wedge \operatorname{gcd}-\operatorname{inv}\left(V, A, a, b, x_{0}, y_{0}\right), \operatorname{Asg}^{b}(\operatorname{subtraction}(A, b, a))\right.$, $\left.\operatorname{gcd}-\operatorname{inv}\left(V, A, a, b, x_{0}, y_{0}\right)\right\rangle$ is an $\operatorname{SFHT}_{\text {of }} \mathrm{ND}_{\mathrm{SC}}(V, A)$.

Proof: Set $i=\operatorname{gcd}-\operatorname{inv}\left(V, A, a, b, x_{0}, y_{0}\right)$. Set $l=\operatorname{less}(A, a, b)$. Set $D=$ $\operatorname{subtraction}(A, b, a)$. Set $f=\operatorname{Asg}^{b}(D)$. Set $p=l \wedge i$. For every $d$ such that $d \in \operatorname{dom} p$ and $p(d)=$ true and $d \in \operatorname{dom} f$ and $f(d) \in \operatorname{dom} i$ holds $i(f(d))=$ true by [6, (23)], [9, (9),(10)].

(19) Suppose $V$ is not empty and $V$ is without nonatomic nominative data w.r.t. $A$ and $a \neq b$ and $A$ is complex containing and for every $d, d$ is a complex on $a$ and for every $d, d$ is a complex on $b$.

Then $\left\langle\operatorname{gcd}-\operatorname{inv}\left(V, A, a, b, x_{0}, y_{0}\right)\right.$, gcd-conditional-step $(V, A, a, b)$, gcd-inv $\left.\left(V, A, a, b, x_{0}, y_{0}\right)\right\rangle$ is an $\operatorname{SFHT}_{\text {of }} \mathrm{ND}_{\mathrm{SC}}(V, A)$. The theorem is a consequence of (17).

(20) Suppose $V$ is not empty and $V$ is without nonatomic nominative data w.r.t. $A$ and $a \neq b$ and $A$ is complex containing and for every $d, d$ is a complex on $a$ and for every $d, d$ is a complex on $b$.

Then $\left\langle\operatorname{gcd}-\operatorname{inv}\left(V, A, a, b, x_{0}, y_{0}\right)\right.$, gcd-conditional-step $(V, A, b, a)$, gcd-inv $\left.\left(V, A, a, b, x_{0}, y_{0}\right)\right\rangle$ is an $\operatorname{SFHT}_{\text {of }} \mathrm{ND}_{\mathrm{SC}}(V, A)$. The theorem is a consequence of (18).

(21) Suppose $V$ is not empty and $V$ is without nonatomic nominative data w.r.t. $A$ and $a \neq b$ and $A$ is complex containing and for every $d, d$ is a complex on $a$ and for every $d, d$ is a complex on $b$. Then $\left\langle\operatorname{gcd}-\operatorname{inv}\left(V, A, a, b, x_{0}, y_{0}\right)\right.$, $\left.\operatorname{gcd}-\operatorname{loop}-\operatorname{body}(V, A, a, b), \operatorname{gcd}-\operatorname{inv}\left(V, A, a, b, x_{0}, y_{0}\right)\right\rangle$ is an $\operatorname{SFHT}_{\text {of }} \operatorname{ND}_{\mathrm{SC}}(V$, $A)$. The theorem is a consequence of (19) and (20).

(22) Suppose $V$ is not empty and $V$ is without nonatomic nominative data w.r.t. $A$ and $a \neq b$ and $A$ is complex containing and for every $d, d$ is a complex on $a$ and for every $d, d$ is a complex on $b$.

Then $\left\langle\sim \operatorname{gcd}-\operatorname{inv}\left(V, A, a, b, x_{0}, y_{0}\right)\right.$, gcd-loop-body $(V, A, a, b)$, gcd-inv 
$\left.\left(V, A, a, b, x_{0}, y_{0}\right)\right\rangle$ is an $\operatorname{SFHT}_{\text {of }} \mathrm{ND}_{\mathrm{SC}}(V, A)$. The theorem is a consequence of (20).

(23) Suppose $V$ is not empty and $V$ is without nonatomic nominative data w.r.t. $A$ and $a \neq b$ and $A$ is complex containing and for every $d, d$ is a complex on $a$ and for every $d, d$ is a complex on $b$. Then $\left\langle\operatorname{gcd}-\operatorname{inv}\left(V, A, a, b, x_{0}, y_{0}\right)\right.$, $\operatorname{gcd}$-main-loop $\left.(V, A, a, b), \operatorname{Equality}(A, a, b) \wedge \operatorname{gcd}-\operatorname{inv}\left(V, A, a, b, x_{0}, y_{0}\right)\right\rangle$ is an $\operatorname{SFHT}$ of $\operatorname{ND}_{\mathrm{SC}}(V, A)$. The theorem is a consequence of (21) and (22).

(24) Suppose $V$ is not empty and $V$ is without nonatomic nominative data w.r.t. $A$ and $a \neq b$ and $a \neq y$ and $A$ is complex containing and for every $d, d$ is a complex on $a$ and for every $d, d$ is a complex on $b$. Then 〈valid-gcd-input $\left(V, A, x, y, x_{0}, y_{0}\right)$, gcd-main-part $(V, A, a, b, x, y)$, Equality $\left.(A, a, b) \wedge \operatorname{gcd}-\operatorname{inv}\left(V, A, a, b, x_{0}, y_{0}\right)\right\rangle$ is an $\operatorname{SFHT}_{\text {of }} \mathrm{ND}_{\mathrm{SC}}(V, A)$. The theorem is a consequence of (16) and (23).

(25) Suppose $V$ is not empty and $V$ is without nonatomic nominative data w.r.t. $A$ and for every $d, d$ is a value on $a$ and for every $d, d$ is a value on $b$. Then $\left\langle\operatorname{Equality}(A, a, b) \wedge \operatorname{gcd}-\operatorname{inv}\left(V, A, a, b, x_{0}, y_{0}\right), \operatorname{Asg}^{z}\left(a \Rightarrow_{a}\right)\right.$, valid-gcd-output $\left.\left(V, A, z, x_{0}, y_{0}\right)\right\rangle$ is an $\operatorname{SFHT}_{0} \operatorname{ND}_{\mathrm{SC}}(V, A)$.

Proof: Set $D_{1}=a \Rightarrow_{a}$. Set $q=\operatorname{Equality}(A, a, b) \wedge \operatorname{gcd}-\operatorname{inv}\left(V, A, a, b, x_{0}, y_{0}\right)$. Set $r=$ valid-gcd-output $\left(V, A, z, x_{0}, y_{0}\right)$. Set $s_{3}=\mathrm{S}_{\mathrm{P}}\left(r, D_{1}, z\right) \cdot q \models s_{3}$.

(26) PARTial Correctness of GCD Algorithm:

Suppose $V$ is not empty and $V$ is without nonatomic nominative data w.r.t. $A$ and $a \neq b$ and $a \neq y$ and $A$ is complex containing and for every $d, d$ is a complex on $a$ and for every $d, d$ is a complex on $b$. Then 〈valid-gcd-input $\left(V, A, x, y, x_{0}, y_{0}\right), \operatorname{gcd}-\operatorname{program}(V, A, a, b, x, y, z)$, valid-gcd-output $\left.\left(V, A, z, x_{0}, y_{0}\right)\right\rangle$ is an $\operatorname{SFHT}$ of $\operatorname{ND}_{\mathrm{SC}}(V, A)$. The theorem is a consequence of (7), (24), (25), and (11).

\section{REFERENCES}

[1] Grzegorz Bancerek, Czesław Byliński, Adam Grabowski, Artur Korniłowicz, Roman Matuszewski, Adam Naumowicz, and Karol Pąk. The role of the Mizar Mathematical Library for interactive proof development in Mizar. Journal of Automated Reasoning, 61(1):9-32, 2018. do1 $10.1007 / \mathrm{s} 10817-017-9440-6$

[2] Adam Grabowski, Artur Korniłowicz, and Adam Naumowicz. Four decades of Mizar. Journal of Automated Reasoning, 55(3):191-198, 2015. doi:10.1007/s10817-015-9345-1

[3] Ievgen Ivanov and Mykola Nikitchenko. On the sequence rule for the Floyd-Hoare logic with partial pre- and post-conditions. In Proceedings of the 14th International Conference on ICT in Education, Research and Industrial Applications. Integration, Harmonization and Knowledge Transfer. Volume II: Workshops, Kyiv, Ukraine, May 14-17, 2018, volume 2104 of CEUR Workshop Proceedings, pages 716-724, 2018.

[4] Ievgen Ivanov, Mykola Nikitchenko, Andrii Kryvolap, and Artur Korniłowicz. Simplenamed complex-valued nominative data - definition and basic operations. Formalized Mathematics, 25(3):205-216, 2017. doi 10.1515/forma-2017-0020.

[5] Ievgen Ivanov, Artur Korniłowicz, and Mykola Nikitchenko. Implementation of the 
composition-nominative approach to program formalization in Mizar. The Computer Science Journal of Moldova, 26(1):59-76, 2018.

[6] Ievgen Ivanov, Artur Korniłowicz, and Mykola Nikitchenko. On an algorithmic algebra over simple-named complex-valued nominative data. Formalized Mathematics, 26(2):149158, 2018. doi $10.2478 /$ forma-2018-0012.

[7] Ievgen Ivanov, Artur Korniłowicz, and Mykola Nikitchenko. An inference system of an extension of Floyd-Hoare logic for partial predicates. Formalized Mathematics, 26(2): 159-164, 2018. doi 10.2478/forma-2018-0013

[8] Artur Korniłowicz, Andrii Kryvolap, Mykola Nikitchenko, and Ievgen Ivanov. An approach to formalization of an extension of Floyd-Hoare logic. In Vadim Ermolayev, Nick Bassiliades, Hans-Georg Fill, Vitaliy Yakovyna, Heinrich C. Mayr, Vyacheslav Kharchenko, Vladimir Peschanenko, Mariya Shyshkina, Mykola Nikitchenko, and Aleksander Spivakovsky, editors, Proceedings of the 13th International Conference on ICT in Education, Research and Industrial Applications. Integration, Harmonization and Knowledge Transfer, Kyiv, Ukraine, May 15-18, 2017, volume 1844 of CEUR Workshop Proceedings, pages 504-523. CEUR-WS.org, 2017.

[9] Artur Korniłowicz, Ievgen Ivanov, and Mykola Nikitchenko. Kleene algebra of partial predicates. Formalized Mathematics, 26(1):11-20, 2018. doi:10.2478/forma-2018-0002

[10] Andrii Kryvolap, Mykola Nikitchenko, and Wolfgang Schreiner. Extending Floyd-Hoare logic for partial pre- and postconditions In Vadim Ermolayev, Heinrich C. Mayr, Mykola Nikitchenko, Aleksander Spivakovsky, and Grygoriy Zholtkevych, editors, Information and Communication Technologies in Education, Research, and Industrial Applications: 9th International Conference, ICTERI 2013, Kherson, Ukraine, June 19-22, 2013, Revised Selected Papers, pages 355-378. Springer International Publishing, 2013. ISBN 978-3-31903998-5. doi $10.1007 / 978-3-319-03998-5 \_18$.

[11] Volodymyr G. Skobelev, Mykola Nikitchenko, and Ievgen Ivanov. On algebraic properties of nominative data and functions. In Vadim Ermolayev, Heinrich C. Mayr, Mykola N1kitchenko, Aleksander Spivakovsky, and Grygoriy Zholtkevych, editors, Information and Communication Technologies in Education, Research, and Industrial Applications - 10th International Conference, ICTERI 2014, Kherson, Ukraine, June 9-12, 2014, Revised Selected Papers, volume 469 of Communications in Computer and Information Science, pages 117-138. Springer, 2014. ISBN 978-3-319-13205-1. doi $10.1007 / 978-3-319-13206-8 \_6$.

Accepted June 29, 2018 